\title{
The Unsymmetrical Space Gravitation Scheme without Time Dimension and Concerning Phenomenon
}

\author{
Gangzhen Jiao \\ School of Environment Science and Safety Engineering, Tianjin Polytechnic University, Tianjin 300384, China
}

\begin{abstract}
Based on the symmetry and unification law, the equivalent interpretation of the relative space-time transformation and the view that time is the artificially defined standard scale, several most important problems were self consistently interpreted in this long paper. It was assumed that all beings, including the quantum field, matter with mass and energy including photon in the universe were composed of space unit (SU) in form of rings. On basis of this, an unsymmetrical space gravity field scheme without time dimension was created. In this theoretical framework and with the existence of entangle rings field around moving matters, constant velocity then is not only the feature of photon but the common phenomenon of all moving matters. That means quantum rings field would be generated by matters motion which is usually explained as equivalence of mass and energy. Thereafter, the unifying of locality and non-locality, the constant velocity of photon and the origin of dark matter could be answered self-consistently.
\end{abstract}

Keywords: Gravity, Photon, Dark matter, Dark energy.

\section{The Essence of Time Led it can't be Included in the Gravitation Field of Matter}

Since the concept of time being created, it has been more than thousands of years. According to the measurement manners of time, it is not hard to see, time is just a physical indicator defined by people accompanied with the necessary of describing the developing or moving speed of things in the world[1,2]. The measurement on matter movement or development relies on the periodical and repeatedly moving of the standard matter like moon, earth, sun or clock in space. Therefore, time is created based on human inspection behavior, responding to the relative relationship of matter movement in space.

A large number of physical facts have confirmed the unity of space and matter. The most immediate and powerful evidence for this comes from the Casimir effect, which confirms that virtual photon in a vacuum quantum field can be converted into real photon, and gamma photon can generate electron-negative pairs, which are the fundamental particles of matter, in a strong Coulomb field. This directly proves that space, energy and matter can be transformed into each other and that means they are unity in nature. When energy like photon is regarded as a special substance without a static mass, it can be said that matter and space are unity in nature. While accompanied with human culture developing, they need to discribe the world more accuratly, as the physical index created in this process, time does not have the innate "first being" parallel with space even surpasses matter. Obviously, there has never been any evidence proving that time can be converted to matter or space.

Our current framework for understanding gravity, devised a century ago by Albert Einstein, tells us that apples fall from trees and planets orbit stars because they move along curves in the space-time continuum. According to Einstein's general relativity, the space-time curve is gravity field. Einstein's general relativity correctly describes the behavior of gravity over close to 30 orders of magnitude, from submillimeter scales all the way up to cosmological distances. No other force of nature has been described with such precision and over such a variety of scales. Although the correctness of the theory of relativity has been proved by a large number of evidences, due to the problems it confronts, people still admit that it is not the ultimate perfect theory. Roughly speaking, in the author's view, as the theoretical cornerstone of relativity, its manner dealing with time, space and matter is also questionable. In particular, it cannot explain a large number of conclusive facts in quantum field, such as nonlocality experimental facts and divergence of gravitational integral. Near the center of a black hole or in the first moments of the universe, Einstein's equation in general relativity breaks. Physicists need a truer picture of gravity to accurately describe these extremes[3].

According to the general relativity, the essence of gravity was posited as the curvature of space-time caused by the mass of matter. However, if we define the natural existence as those not relied on human investigation consciousness, and regard it as the "first existence", the above view of gravitation is essentially endowing time the "first existence" which is higher than matter and parallel with space. Obviously, if gravity is the curvature of space-time by matter mass, as shown in Figure 1 (a), it also means that flat 4-D space-time can exist without matter mass (as shown in Figure 1 (b)). In this way, it is clear that time is more innate than matter, and more consistent with space than matter. Obviously, this is contrary to the above fact that matter and space can be intertransformed.

Moreover, the gravity scheme in general relativity gave mass higher existence priority over gravitational field which resulted in the separation of mass from the gravitational field. On one hand, this led to disable of the scheme with infinite space-time curvature in infinitesimal particle or black hole conditions, and this was the theoretical consensus now, on the other hand, it also contradicted with the universal gravity law in which mass could not be separated with gravitational field. 
Therefore, it is logical to propose a logically feasible gravity

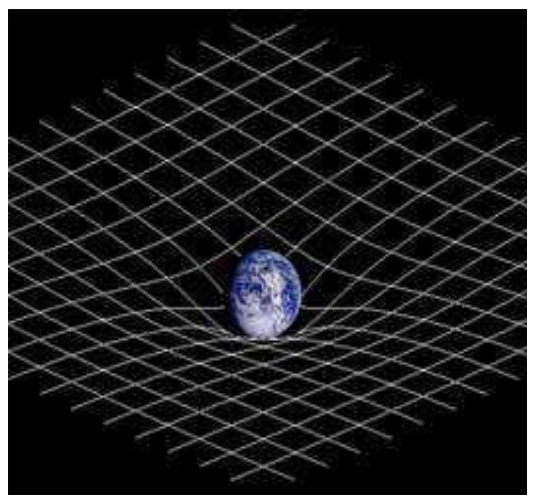

(a) solution that does not have these problems.

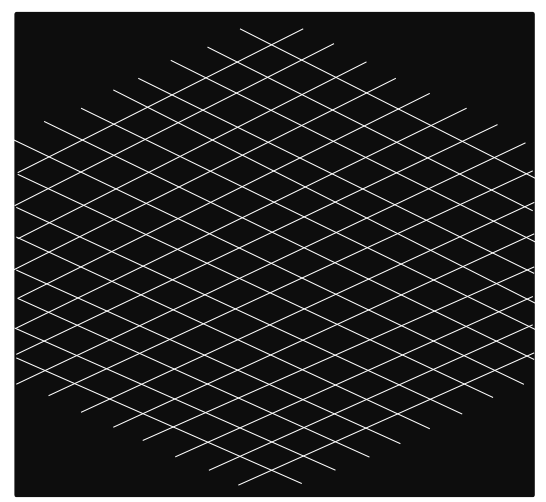

(b)

Figure 1: Space-time bending caused by matter mass (a) and flat time and space without matter (b)

We can also intuitively analyze the logic rationality of the space-time curved gravity scheme of general relativity. According to the scheme, the space-time curvature is caused by the mass increasing with the nearer distance from the material as shown in Figure 1 (a). But why does the object matter accelerate approach in the direction that the curvature of the space-time of the subject matter increases so that gravity occurs? Could the gravitational field really be described as a space-time web in which it bends downward more as gets closer to the subject matter, and the bigger the curvature, the object matter is more likely slide down to the lower depth of the space-time web of the subject matter? If this is the case, the process obviously depends on the object itself being gravitated, that is, on its mass and on a third mass below it. How does the third matter underneath gravitationally interact with the object matter? This is obviously an infinite loop with no solution. Therefore, logically, the relativistic space-time curved gravity scheme can not provide a complete self-consistent explanation of gravitational action without relying on the existence of background gravity and mass.

Based on the innate and artificially defined dimensional essence of time, and the cognition that matter and space are unitary inherent existences, the following problems should be solved: the order launched by typical relativity that Lorentz transformation of space based on the time transformation firstly has to be revised. It can only transform space firstly, and then lead to the transformation of time; secondly, since time lost the innate existent priority to matter, naturally it can't form the four-dimensional space-time and express the formation of gravitation by matter mass. Accordingly, the mechanism of gravitation also needs to have a new expression form in which time should be excluded.

\section{2. "The Law of Symmetry and Unification" Should be the Most Fundamental Law Governing Everything in the Universe}

\subsection{Why it Should Be Most Fundamental Law?}

There has long been searching for the most fundamental laws governing the universe, but so far there is no clear answer. As we all know, Galileo, the founder of classical physics, proposed that the laws of mechanics were invariant in all reference systems, and Einstein furtherly developed it and proposed that all physical laws were invariant in all reference systems. From Galileo to Einstein, they are essentially the deepening and developing process of the understanding that the physical laws have unity and symmetry when we investigate objects in all reference systems. Specifically, it has two meanings. On one hand, it refers to the unity of physical objects following physical laws in all reference systems. The invariance of the form of mechanical laws and all physical laws is the embodiment of unity. On the other hand, all the physical objects in the reference system are equal and have the same weight when they follow the physical laws, that is, the embodiment of symmetry. The concrete manifestation of symmetry is the equivalence and equality of all physical existence in the reference frame. In Galileo's view, the laws of mechanics are symmetrical, unified and equal when examining physical objects in all reference frames. While in Einstein's view, all physical laws have equal weight and symmetrical unity when examining physical objects in all reference frames. Therefore, the two stages are actually the finding process on the physical objects in all reference systems following the law of symmetry and unity (short in symmetry law below). In common sense, further reasoning along the logic of the two most recognized and success pioneers of physics is most likely to get the most fundamental law. It should be considered that on this basis, it can be furtherly expanded. That is, not only all physical laws, but all physical existence should follow the symmetry law.

In fact, not only in Galileo and Einstein's theory, the fundamental governing role of symmetry law is reflected in many aspects in the physical world. It is believed that the superluminal connection of particles with quantum entanglement is based on the conservation of angular momentum. CPT symmetry is the most fundamental principle that modern physics theory research follows. According to Knott's theorem, conservation inevitably comes from a kind of symmetry, and the three most fundamental conservation laws are derived from a kind of symmetry respectively: space translation symmetry leads to momentum conservation law, time translation symmetry leads to energy conservation law, and space rotation symmetry leads to angular momentum conservation law.

Moreover, Tsung-Dao Lee once said that "asymmetry is the basis for the existence of physical indicators". Lee's view implies that the fundamental feature of all existences is asymmetric. For instance, it's only able to write clearly on a blackboard with those not black chalks. However, according 
to the author, its opposite meaning, that is "symmetry is the basis of non-existence of physical indicators and even the matter" makes the most important and extraordinary fundamental significance, because it can lead us to attend to the concrete form of the most fundamental law. Just as we can't write clearly on blackboard with the same black chalk for it has no difference with the background. Absolute symmetry is the only feature of "emptiness". Because there is no difference in all aspects in it, the essence of it is absolutely uniform and equal. One hand, emptiness is the most basic exist in the universe, and on the other hand the unity of the universe determines that all exists should follow the same law as "emptiness". Specifically, it means that the symmetry law embodied in "emptiness" will run through the existence, operation and evolution of all beings and all of them are equal, and symmetry in the universe. Nothing could be unique or superior on others. This ratiocination is accordance with Galileo and Einstein's reference. Both mean that the symmetry law is the most basic law running the universe. In details, for unity defines the scale of the law working, while symmetry defines the content of it, so symmetry has more specific influences than unity. In physical views, symmetry can be thought to be embodied in two aspects: one is embodied in the physical index itself. All values of the index have the equal exist potence in the space; secondly, all the physical existences have equal potence in space distribution and interaction. Of course, there should be many kinds of details resulted by symmetry law. Findings of symmetry as CPT, Galileo and Einstein's, even other theories are only parts of them. It can be found that, along this direction the main current physical problems, especially those concerned with gravity, can be reasonably explained self-consistently. All that remains is to refine the details along this direction.

Through analysis, the relationship of symmetry and asymmetry can be summarized in the following aspects: (1) The maximization of symmetry is the ultimate goal of the evolution and motion of all matter in any systems of space; (2) Asymmetry is the fundamental driving force of the evolution and motion of all matter in any systems of space; (3) The total amounts both of symmetry and asymmetry are always conserved in the whole universe[2,4,5].

\subsection{Construction of the Most Basic Unit of All Things based on the Symmetry Law}

First of all, based on the unity of the universe, it should be clear that all things should be composed by the completely same basic unit. Because the free state of the unit is in the space, it can be named as space unit ("SU" for short). SU is posited not only the basic unit of quantum field, but also the basic unit of matter with mass and special matter with no static mass, such as energy like photon.

Different values of physical index have the same weight equality for existence is the basis to form the configuration of SU. According to the rule of symmetry law, it is inferred that the SU should has a relationship similar to the space volume $(V)$, mass $(m)$ and space density $(\rho)$ of matter $(\rho=m / V)$. Since it has no mass, the corresponding $m$ is the number of SU " 1 " and it's a dimensionless number. Based on the symmetry law, there should be no difference in all directions of spatial density. The equal space density points on all direction should be a three-dimensional (3-D for short) symmetrical spherical surface around the core and the sum of the density on all spheres are constant. If the radius of the sphere is assumed to be " $R$ ", then, by inference from the symmetry law, $R$ should be distributed between unlimited approaching 0 and unlimited approaching infinity, that is $R \in(0, \infty)$ (excluding zero and infinity which do not exist actually), and the corresponding volume is spherical surface $V=4 \pi R^{2}$. Then, the space image (shorted in SI) density at $R$ should be $\rho_{0}$ is $1 /\left(4 \pi R^{2}\right)$. On the other hand, a 3-D spherical surface can be considered as a 2-D circle symmetrically exists on all angular of planes on basis of symmetry law. Therefore, the sum of the density at distance $R$ can be considered as a 2-D circle. Its volume is $2 \pi R$, and the corresponding spatial density $\rho_{0}$ is $1 /(2 \pi R)$. Through the fitting analysis of asymmetric gravitational field with Newton's gravity formula $\left(F=G M m / R^{2}\right)$, the circle scheme is more reasonable. The latter structure of 2-D circle of the relatively stationary SU is shown in Figure 2[1,2].

Figure 2: Relatively stationary structure of space monomer

$\rho=1 /(2 \pi R)$ can be deformed into $\rho R=1 /(2 \pi)$. This expression is of vital importance in the processing of the density of the outer SI of relative moving matter and SU below. Reasoning from the symmetry law, SU and matter composed by it should all meet this formula. Then the following important inference can be obtained: the density of the space at a point outside the matter is only related to the distance from the point to the matter, and is independent from the relative state of motion. Specifically, the product of the space density and the distance is always constant " $1 /(2 \pi)$ ".

In order to differentiate the exclusive space from the common space of background, it can be named as SI. For as $R$ approaches 0 infinitely, the core of SU with infinite space density is the main body of matter, it is named as object image (shorted in OI). It should be noted that, although OI has an infinite density value, as to the specific small SI, it still has a specific density value. So, essentially the nature of OI is still SI. This lays a foundation for the formation of 2-D ring of matter with static mass, and 3-D twist closed ring energy without static mass in the frame of symmetry law.

\section{Rings Formed by SU Through Quantum Entanglement}

In view of the fact that the correctness of relativity has been proved by a lot of evidences, and on the other hand, relativity theory is facing the dilemma of theoretical breakthrough. With the precondition that time is non-inherently objective 
exist as space, the author agree with the Lorentz transformation results of space and time. However, at the same time, it was insisted that the process of it should be revised. Specifically, under the guidance of the artificial standard ruler nature of time, accompanied with relative motion, the transformation order of relative space and time should be interpreted in a different manner.

The classical space-time transformation form of special relativity is shown in Figure 3 (a). In the stationary reference frame $\mathrm{O}$, the illuminant is stationary relative to the observer $\mathrm{A}$, and the moving reference frame $\mathrm{O}^{\prime}$ has velocity $\mathrm{v}$ along the $\mathrm{x}^{\prime}$ axis relative to A. As $t=0$, the origin point $\left(x=x^{\prime}=0\right)$ of the two reference systems coincide. In both reference frames, the illuminant emits photons to the observer $\mathrm{A}$ at the origin. According to special relativity, A has the same time and space as the relatively stationary illuminant $\mathrm{O}$. Suppose the time of photon arriving at $\mathrm{A}$ from the illuminant at $\mathrm{O}$ is $t_{0}$, and the space distance between $\mathrm{O}$ and $\mathrm{A}$ is $\mathrm{S}_{0}$, then, $S_{0}=c t_{0}$. When the photon from source at $\mathrm{O}^{\prime}$ to $\mathrm{A}$, it appears to A that the photon passing through the space and time are same with reference frame O', which can be named $S_{v}$ and $t_{v}$ respectively. On basis of the constant value of speed of photon, $S_{v}=c t_{v}$ could also be deduced. More details concerning on this won't be introduced because the whole derivation is too complicated. What can be explicated is that the special relativity is based on the premise that the time of photon and illuminant is consistent. Then the relative time $\mathrm{t}_{\mathrm{v}}$ can be firstly deduced as this[6]:

$$
t_{v}=t_{0} /\left(1-v^{2} / c^{2}\right)^{1 / 2}
$$

On this basis and $S_{v}=v_{v}$, the relative space $S_{v}$ is obtained:

$$
S_{v}=S_{0} /\left(1-v^{2} / c^{2}\right)^{1 / 2}
$$

Following the same principles with relativity including physical equations of different inertial systems have the same form, the speed of photon is constant, and photon and its source have the same time, the above derivation process can be simplified as shown in Figure 3 (b). In the view of observer A, except that the photon from $\mathrm{O}$ is moving along the straight line of OA, the photon emitted from $\mathrm{O}^{\prime}$ is also moving along the straight line of O'A. Combined with the knowledge of plane geometry, it can be known that $\triangle A O O^{\prime}$ is a right triangle, and the Pythagorean theorem is satisfied as follows: $\left(c t_{0}\right)^{2}+\left(v t_{v}\right)^{2}=\left(c t_{v}\right)^{2}$. According to this relation, Eq. (1) can also be derived. Combined with $S_{v}=c t_{v}$, Eq.(2) can be obtained.

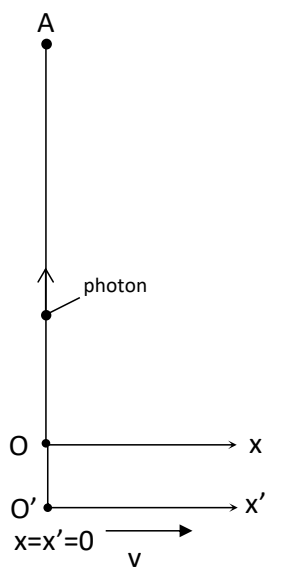

(a)

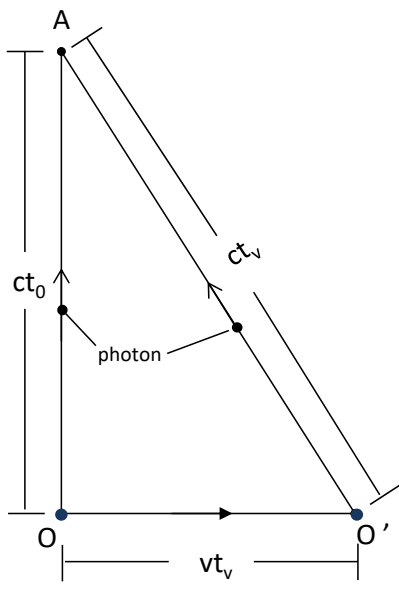

(b)
Figure 3: The original reasoning (a) and equivalent reasoning manner(b)[2]
According to the principle that non-basic being consists of basic being, thus the basic being couldn't be expressed by the non-basic being. On the premise of negating time has the first innate existence and accordingly it does not have the feasibility to be transformed firstly, it is necessary to provide an equivalent explanation scheme in which relative motion directly leads to the transformation of space instead of through time transformation firstly. The derivation form of relative space-time transformation introduced in Figure 3-1(b) is essentially same in nature as the classical derivation form of relative space-time transformation. In essence, both of the above two derivation processes are based on the premise that time has innate first existence, and time can be transformed firstly. And then it leads to the relative transformation of space. While, the derivation form in Figure 3-1(b) provides convenience for transformation of space at the first and then leading to time transformation. This form accords with the property that time is artificially defined as a scale based on the movement of matter space, and without the feature of first innate existence.

\subsection{Equivalent Interpretation of Relative Space-time Transformation based on Spatial Transformation at First}

Moreover, according to the general theory of relativity, the nature of gravitational field is the curvature of space-time caused by the mass of matter. In view of this and the fact that time cannot participate in the formation of gravitational field, there should be an exclusive epitaxial space of matter with decreasing density along with the distance outside the matter which is also accordance with the law of the universal gravitation. The space motion of any matter is in the epitaxial SI of other matter (the epitaxial SI of matter does not occur directly by its SUs, see the related analysis below), and the gravitational action occurs through the epitaxial SI. Then, the relative transformation of space can be explained from the perspective of the changing of SI density due to relative motion but not through the non-first existence time transformation (as shown in Figure 4).

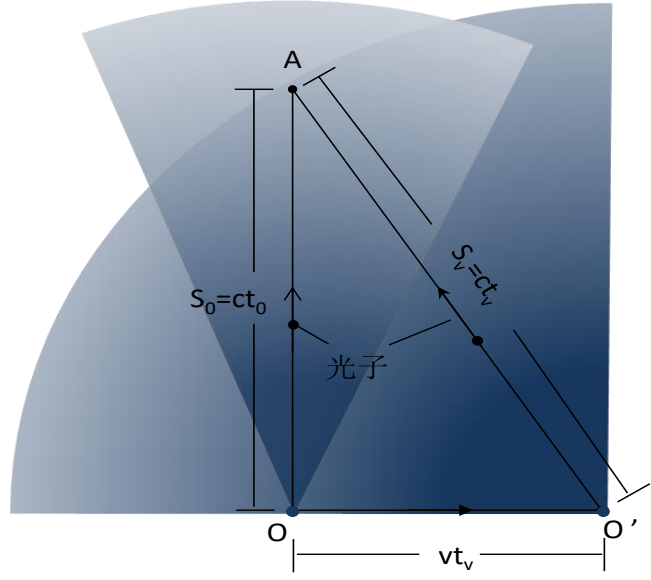

Figure 4: Schematic diagram of space change with SI density[2]

It was supposed that the observer at A is stationary relative to the illuminant at $\mathrm{O}$, and the relative epitaxial SI of the illuminant at $\mathrm{A}$ is $\rho_{0}$. From photon left $\mathrm{O}$ until it arrived at $\mathrm{A}$, relative stationary makes that the relative SI value of illuminant at $\mathrm{A}$ is always $\rho_{0}$. When the illuminant move with speed $v$ relative to the observer at $\mathrm{A}$, as photon leaving the source at $\mathrm{O}$ its relative density to observer at $\mathrm{A}$ is still $\rho_{0}$, and 
accordingly the distance between them was also still $S_{0}$. It's like taking a photo as photon just leaves O. However, at the moment the photon arrives at $\mathrm{A}$ the illuminant has moved to $\mathrm{O}^{\prime}$, and their distance then is $\mathrm{AO}^{\prime}\left(\mathrm{S}_{\mathrm{v}}\right)$. The relative SI density of illuminant at A will decrease to $\rho_{v}$. According to above analysis, and on basis of symmetry law, the relationship of SI and distance of all grades of matter have the same forms with $\mathrm{SU}$, that is, the product of the outside SI density and its distance to the matter is constant: $\rho S=1 /(2 \pi)$ (here the $R$ above is symbolized by $S$ ), and it equals to: $\rho_{0} S_{0}=\rho_{v} S_{v}$. Furthermore, there are $t_{v}=S_{v} / c, S_{0}{ }^{2}+\left(v t_{v}\right)^{2}=S_{v}{ }^{2}$, by combination of the three formulas, the relative motion SI density $\rho v$ can be obtained as following[2]:

$$
\rho_{v}=\rho_{0}\left[1-(v / c)^{2}\right]^{1 / 2}
$$

In combination with $\rho_{0} S_{0}=\rho_{v} S_{v}$, the relative space transformation Eq.(2) caused by relative motion can be obtained. Combined with $S_{v}=c t_{v}$, Eq.(1) of the relative time transformation can be obtained.

In this way, the relative space-time transformation form is derived from the transformation of the space firstly. In this derivation, time transformation is only a superficial phenomenon caused by space transformation. This is consistent with the artificial scale essence of time. Correctness of the derivation sequence is of great importance. This is not only related to the correct understanding of the nature of the relationship between matter with space and time, but also related to the correct construction of the mechanism of matter mass and gravity. When the time dimension is excluded from the gravitational field, the mechanism of gravity forming can be directly attributed to the epitaxial SI density of matter based on the symmetry law.

However, there are two crucial problems need to be solved. Question 1: according to Lee's reasoning that the asymmetry will lead to the observability of physical indicators, the SU with decreasing external density which actually is asymmetric structure should be observed. While till now all forms of SU are still not directly detected? Question 2: if as mentioned above, the matter represented by the illuminant has an epitaxial SI, is this SI the superposition of all the SU composing the matter? If so, it cannot explain the fact that the SU has not been detected. If not, how does the matter epitaxial SI form? The answers to these two questions are with crucial importance for they are related with the forming mechanism of matter with SUs and the correctness of all the assumptions.

Through analysis, it is found that the mechanism of the undetectable feature of SU can be solved from Eq.(3). According to the theory of relativity, the speed of matter with mass cannot increase to the speed of photon, but can only be infinitely close to it. However, in order to explore the source of the undetectable feature of SU, it can be assumed that the velocity of the illuminant can be increased to c relative to the observer as Eq.(4). It can be certified below that the SU always has the velocity of $c$ [2]

$$
\lim _{v \rightarrow c} \rho_{v}=\lim _{v \rightarrow c} \rho_{0} \sqrt{1-\left(\frac{v}{c}\right)^{2}}=0
$$

From Eq.(4), it can be found that the density of the epitaxial SI of SU relative to the outside world will always be 0 and independent with the distance to it. According to "symmetry means undetectable", it can be proved that the SU, no matter it composing matter, photon or in free state, will always be undetectable, and this is independent from the advanced extent of observing instruments. Then, it is necessary to prove the SU always move at the speed of photon. It can be certified as shown below. Due to the most basic unit property of SU, it cannot be affected by any force, so its speed must always be a constant, which is assumed to be $x$. It was assumed that a SU moves back and forth between two plates with a distance of $\mathrm{L}$ in the stationary reference frame. It can be gained from this stationary reference frame that:

$$
t_{0}=2 L / x
$$

When the two plates moved right with a speed of $v$, on one hand, the time of the moving reference frame is no longer the static time. According to the theory of relativity, it can be gained that:

$$
t=t_{0} /\left(1-v^{2} / c^{2}\right)^{1 / 2}
$$

On the other hand, the velocity of SU would not be affected by the movement of the reference frame. In the vertical direction, it completes the round trip between the two plates with the projected velocity $\left(x^{2}-v^{2}\right)^{1 / 2}$. Then it can be gained that:

$$
t=2 L /\left(x^{2}-v^{2}\right)^{1 / 2}
$$

From Eq.(5), (6), and (7), it can be gained that:

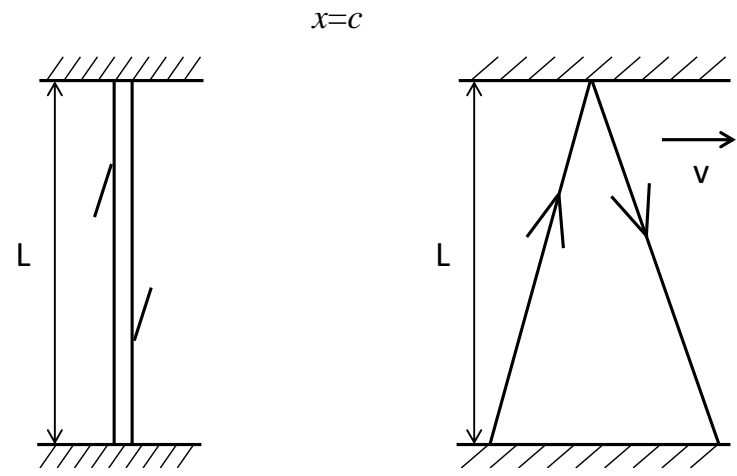

Figure 5: Proof on the velocity of SU is constant to the speed of photon

Thus, the origin of the undetectable feature of SU is solved. In addition, it can also be speculated that the relative space distance between an SU and any position outside will always be infinite (as shown in Eq.(8)):

$$
\lim _{v \rightarrow c} S_{v}=\lim _{v \rightarrow c} S_{0} / \sqrt{1-\left(\frac{v}{c}\right)^{2}}=+\infty
$$

Accordingly, it can be inferred that although SUs constitute everything, no matter the actual distance how close to it, it would have infinite relative distance. Therefore, there is no possibility for collision or mergence between them and the total number of $\mathrm{SU}$ is conserved in the universe. Being capable of infinitely close to SU also means that, it's unable to measure the density or number they are in unit space or matter.

\subsection{Rings are Formed by SU based on Quantum Entanglement}

Since the velocity of SU is constant of c, and its external density is always zero, next point we need to know is the 
generation mechanism of external SI of matter, which is also the basis of gravity field. As is known to all, according to the quantum field theory, the space is full of quantum. Energy and mass are regarded as the excited state of quantum field. In order to solve the integral divergent of gravitational field caused by the infinitesimal point of particle matter model, string theory proposed that the basic unit of matter is closed string with a finite large diameter, and the basic unit of energy is open string. In other words, closed and open strings are considered to be the most fundamental units in the universe. However, this view violates the fact that energy and mass are interchangeable and also inconformity with the general premise that the universe is unity. Based on the premises that SU formed everything, localized reality features of macro phenomenon and unlocalized reality features of micro phenomenon should be unified, and inspired by string theory and the quantum field theory, the author thinks that matter with mass and energy including photon should both be closed-rings with quantum property composed by SU based on mapping entanglement as shown in Figure 6[7,8].
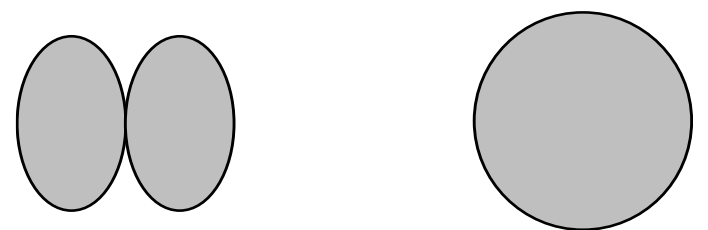

Figure 6: 3-D twist ring of photon and closed-ring of matter unit[2]

The 2-D closed ring of matter with mass is the basis of its mass and asymmetric gravitation field. The open string is inferred to be approximate open ring in 2-D plane and actually is twisted closed ring in 3-D. It is formed by the symmetrically distribution of the object ring on all planes through the axis of photon motion on basis of the symmetry law. This automatic manner guarantees the completely symmetry of OI and SI by orbiting on each other and it also resulted into the no static mass feature of photon. In contrast, the 2-D ring is asymmetry for them especially for SI. This resulted into the mass and gravitation effect of the steady unit. What need to be highlighted is, both of the 2-D ring of matter and 3-D ring of energy are mapping entangled together of more than one SU. The mapping entanglement of SUs means that, on one hand, all the SUs composed the ring entangled with each other and they are equivalent to be detected as excited quantum field energy or particles. This is assumed to be the origin of uncertainty phenomenon. In other words, the so-called nonlocality is not the super velocity existence of a single particle in space, but the existence of multiple SUs which have completely same features except position in space. On the other hand, once the motion state of wave function of one SU is disturbed, the states of wave function of other SUs also disappear at the same time. For any one of them can be active in state of the energy like photon or the particle matter like electrons, based on the symmetry law, only one of them can be detected as protagonists at any time point. In other words, as the wave function state of any one of the SU composed the ring is disturbed, the whole wave function will collapse and other SUs will recover to the free states, in which they all can't be detected as Eq.(4). Obviously, at the same time all the energy and mass of the wave (ring) would be completely carried by the detected one.
The reasons why photon is not only regarded as string like string theory can be summarized as below: (1) The idea of string conflicts with the transformation of photon and matter; (2) The string concept is difficult to provide a reasonable explanation for why the open string breaks and how SU will exist at the breakpoint; (3) The concept of assuming photon as string and mass as closed string cannot self-consistently explain the no static mass behavior of photon. (4) It is a reasonable consequence of the symmetry law to treat the photon as a twist 3-D ring.

Moreover, it should be inferred the quantized features of beings composed by SU based on mapping entanglement are not microscopic special cases, but the universal and the key fundamental relationship that supports the existence and development of all things in the universe. It is believed by the author that the nonlocality is the more essential and fundamental basic relationship between all things in the universe, and the locality is only a false appearance.

Above deduction can be testified through the delay observation experiment of double-slit interference of mono electron. It is found that the wave interference phenomenon of the mono electron will disappear as observed, and it will occur when the electron is not observed. In common sense and classical physical theory, mono electron can't pass through two slits at the same time and also can't have wave expression for electron motion is not entangled with SUs in the space. Obviously, classical theory cannot explain above phenomenon. Moreover, studies have found that not only electrons, but also protons, neutrons, and even more massive soccer alkenes have alike double-slit phenomenon that demonstrate their wave-particle duality. Thus, it is reasonable to propose that, based on the symmetry law, not only photon and electrons, but all other macroscopic matter motion can form quantum entangled rings field with SUs in space.

Take the electron double-slit interference results for instance, it should be thought that except the moving electron, there are also mapping images formed by SU and they are entangled with the subject image electron to form waves (rings). When they pass through the double slit, the entangled rings are divided into two parts as others described. However, for the rings are composed by independent SU which ensures their entanglement relevance and their interference relation won't be disturbed in this process. But, as anyone of mapping or subject images is observed, the two parts of the entangled ring will collapse meanwhile. For the mass and energy of the electron is equivalent to the entangled ring, the ring can collapse and convert into electron as any images on it is excited by detecting. This should be the mechanism of uncertainty of the electron. This explanation is feasible explanation for the double-slit interference results. This also supports the inference that the entanglement rings field will be formed when macroscopic massive matter moves. At the same time, it also means that behind the locality of macroscopic matter, there is also the unlocalized reality. The reason for their locality expression is the too massive mass of the subjects compared with the energy of their rings. It should be said that because electromagnetic, strong and weak forces have not fully involved in the most fundamental nonlocality relationship, they can be established on the basis of locality principles. While as to the gravity which is directly based on 
the most fundamental locality, then the front physical theory can't work through any more. Moreover, this deduction can also be the basis for understanding the gravitational effect of dark matter and constant velocity of photon. Specifically, the dark matter mass phenomenon is the inevitable reflection of the macroscopic nonlocality nature.

\subsection{Formation Mechanism of the Exclusive Epitaxial SI of Matter}

These years most attention has been paid to the uncertainty of photon or electron in the transverse direction[7,8], but the influence of uncertainty on the propagation direction of photon is not considered as shown in Figure 7.

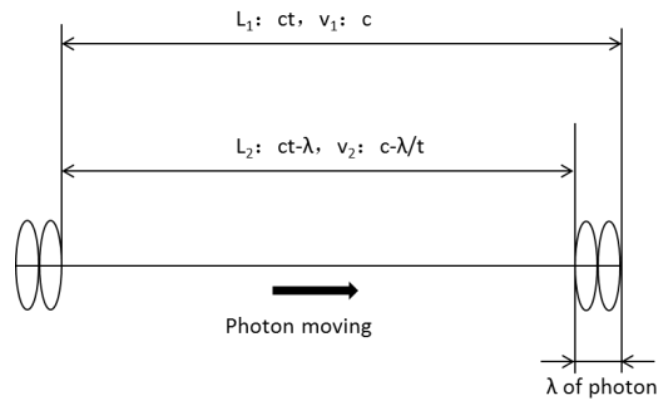

Figure 7: The photon velocity when consider uncertainty caused by mapping entanglement[2]

If supposing the elapsed time of the photon from the illuminant to the observer is $t$, and the corresponding velocity $v_{l}$ is $c$, then the distance $L_{l}$ is ct. Since the uncertainty feature in transverse of photon is originated from the quantum entanglement SU ring, it can be ratiocinated that the uncertainty feature should also be reflected in the direction of photon motion. The velocity of SU would be affected by the wave length of the photon. The quantization feature of photon will make the position of SU at the wave tail equivalent to the $\mathrm{SU}$ at the wave head. Specifically, the wave-tail SU at a distance of $L_{2}\left(L_{2}=L_{1}-\lambda=c t-\lambda\right)$ would be equivalently observed because the wave-head SU actually has been observed which resulted into the photon wave collapse. It can be found that the wave-tail SU of photon actually traveled one wavelength less while it is equivalently observed. If the velocity of wave-tail SU is defined $v_{2}$, then $v_{2}=c-\lambda / t$. This means that the quantized nature of the photon will make the wave-tail SU actually show a slightly less speed than $c$. Since photon and matter essentially both are 3-D closed rings formed by mapping entangled of SUs, the external SI of matter with mass can be considered to occur also by the quantized nature of the $2-\mathrm{D}$ closed ring. The SU which composed the 2-D closed ring with a diameter of $d$ in matter would have relative common SI density as shown in Eq.(9). Moreover, from Eq.(3-9) the density of common SI would decrease following the distance increasing to the ring as shown in Figure 8[2,4].

$$
\rho_{v}=\left.\rho_{0}\left(1-v^{2} / c^{2}\right)^{1 / 2}\right|_{v=c-d / t}>0
$$

The premise for ensuring Eq.(9) making sense is $t \geq d /(2 c)$. It actually means that in order to ensure the SI density greater than zero, the position focused can't be inside of the closed ring. Once it's inside of the ring, $\rho_{v}$ would lose its meaning. This shows that the existence of the quantized feature of the closed ring is indeed directly related to the observer's position and accordingly observation behavior. According to the results of double slit interference delay observation, if the observation behavior occurs on the ring and it terminates the motion on the ring of any SU, the ring (wave) will disappear directly based on the symmetry law. If the observer entered the closed ring, the SI would not exist to the observer due to the failure of Eq.(9). As to the 2-D ring with mass, it can be inferred only when the observer is outside the ring and the entanglement state is guaranteed, the quantum properties of the ring and the gravitational field can exist. From the symmetry law it can be inferred that the ring matter formed by SU has the similar epitaxial SI and the quantized feature as SU, and this is of crucial importance and fundamental influences. Figure 8 shows the ratio of outside common relative SI $\left(\rho_{v}\right)$ of a 2-D ring to the relative static $\operatorname{SI}\left(\rho_{0}\right)$ of SU following $x$. On basis of Eq.(9), the ratio $y$ is $\left.\left(1-v^{2} / c^{2}\right)^{1 / 2}\right|_{v=c-d / t}=$ $\left(2 d /(t c)-(d /(t c))^{2}\right)^{1 / 2}$ and $x$ is the product of time and the velocity of photon (c) which present the distance from the site to the ring in unit of the diameter $(d)$ of the ring. Specifically, for the focused site can't be inside of the ring and meanwhile it's necessary to ensure Eq.(9) making sense, so it need to meet $t \geq d /(2 c)$, and the minimum $x$ should be $0.5 d$ and accordingly the $y$ is 0 . This trend should be the origin of asymmetry gravity field which meets the law of gravity. Moreover, since $\rho_{v}$ is the inhomogeneous SI part which forms the asymmetric space gravitational field (see the description of the gravitational field structure below), thus this deduction is also the basis for explanation on the integration divergence of the gravitational field problem of infinitesimal matter particle.

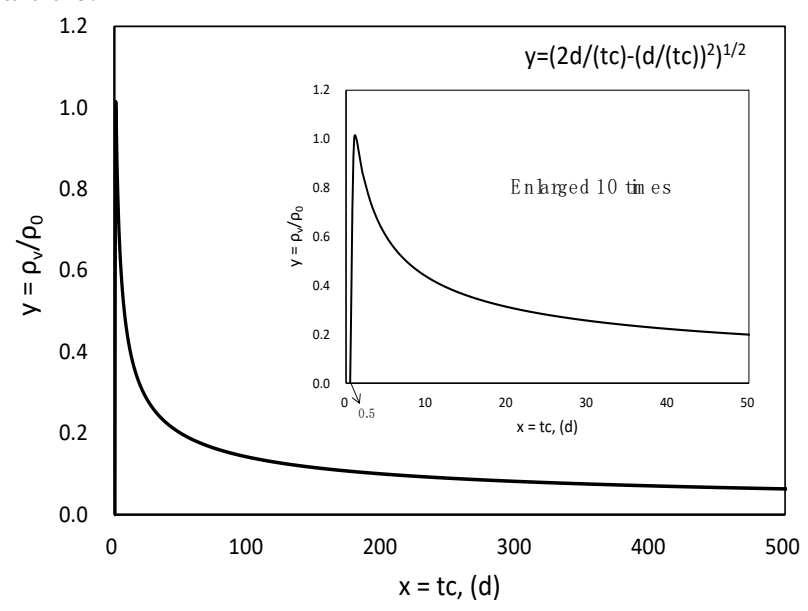

Figure 8: The common SI density outside the ring

\subsection{Analysis on the Formation Mechanism of Closed Ring}

It should be said, the observable physical being can be classified into two categories. One is based on the interaction between the four fundamental forces. They have features of localized reality and theirs speed are limited by the velocity of photon; the other is the physical existence with very little mass or no mass at all. Their motions have the feature of uncertainty, in other words, nonlocality features. The motion of locality beings are assumed on the basis of four fundamental forces, and their action mechanisms are uniformly assumed to be the exchanging of medium with mass or energy by the gauge field. As to the motion of the nonlocality beings, the spatial distribution probability are mainly described on the Schrodinger equation and uncertainty law, and there is no universally accepted explanation on the mechanism. 
From this theory, on the requirement of the symmetry law, the uncertainty is speculated to be the symmetrically distribution of the mapping entangled SU ring and its higher states on all planes. However, during the composing process of the mapping entangled 2-D or 3-D ring, as the most fundamental unit, SU should not be operated by the four fundamental forces. SU formed the bulks of all four kinds of fundamental forces, thus they don't have any mass, energy, charge or color charge. As the most basic unit, not only the four fundamental forces can't affect SU, but also all background would not have any effect on them.

Mapping entanglement is the basis of the quantization feature of the rings. Through analyzing, the mapping entanglement relation should only be established on the symmetry law and the relativity of motion. However, the relativity of motion is also based on the symmetry law. That means, when an SU moves towards another, even if the latter is stationary in that direction, it is impossible to distinguish which move firstly. If we want to break the opposite motion states of them, the motion of either SU stopped is equivalent to the other. The motion state of any SU on the ring is stopped by being observed, and then the wave function of the ring would collapse completely. Therefore, based on the relativity of motion in compliance with the symmetry law, SUs can form mapping entangled rings. This guarantees all SUs composing the 2-D or 3-D rings share the same space positions and motion function. This is the main reason of the uncertainty and nonlocality expressions of quantum particles. On the basis of Eq.(9), common SI above 0 can be formed. In essence, the relativity of motion also occurs on the basis of the symmetry law, that is, the two SU with same features are equal and symmetric in motion.

However, simply moving in opposite directions on the same line is not enough to explain why they constitute photon or matter rings. In the construction process of SU structure, it can be found that the essence of OI is also SI but with infinite density. The two should have equal orbiting authority in compliance with the symmetry law. In a single SU, the OI is always orbited by its outer SI. Obviously, the outer SI of a SU cannot be orbited by its own OI at the core. While as the common SI is formed by SUs, under the requirement of the symmetry law, they are possible to orbit the common SI then. What should be noted is, as common SI is generated in Eq.(9), the diameter used is the space distance between the two SU. Since they do not yet form a real ring, they can be named spurious ring. Thus it can be posited that the real ring formed by SU around the common SI is based on the existence of spurious ring. Moreover, since there are infinitely numbers of SUs in front of the moving SU, and the velocity of SU motion will not decay forever, so there should also be infinitely numbers of SUs that can form mapping entanglement rings with it. The number of rings caused by the SU movement is accordingly infinite. More analysis find that this inference can be testified each other by the constant velocity of photon and the unity process of local and nonlocality.

\section{Mass and Gravitational Field Generation Mechanism of 2-D Closed Ring}

As the most fundamental law, the symmetry law should govern the occurrence of mass and gravitational field also.
The following two points are considered in designing the mechanism: (1) The photon which was regarded as 3-D twist closed ring and 2-D approximate string has no static mass; (2) While the 2-D closed ring, which was assumed to be the basic unit of matter, has mass and gravitational field. Thereafter, it can be inferred, whether there is 2-D closed ring in a plane is the key to create mass and gravitational field. Combined with the symmetry law, it is found that the existence of 2-D ring decides whether the OI and SI can symmetrically orbit on each other. The basic inferential framework for the creation of gravitational field and mass is that, the existence of 2-D closed ring only makes the inner-ring SI completely orbited by OI, while the outer-ring SI is not symmetrically orbited by the OI, which would make SI outside the ring asymmetry (or symmetry breaking) relative to the inner-ring SI. As a whole, the SI of inner and outside the ring would have asymmetry and the asymmetry of SI is the actual essence of curved space-time gravitational field in general relativity after stripping time dimension. In this frame, the static mass actually corresponds to the SI surrounded by the 2-D closed ring. When there is other 2-D closed ring outside the ring, the density of its outer SI decreases with the increase of distance. In compliance with the symmetry law, under the requirement of maximally compensation on its SI asymmetry, the gravitational action express satisfying Newton's law would occur. Therefore, the essence of gravitation satisfying the universal law of gravitation should be summed up as the process of maximally compensating for the asymmetry of SI of the two acting matter.

Combined with the understanding of the inner unity of philosophy, mathematics and physics, 0 and natural numbers are quoted to describe the homogeneity property of SI inside and outside the OI ring. 0 and the even numbers 2, 4, and 6, etc. represent orbits of SI inside and outside the ring with uniform density, while the odd numbers 1,3 , and 5, etc. represent the orbit of the SI outside the ring with uneven density. Mapping entanglement not only endows the ring with integrity, but also makes the SI in the ring have uniform density, so it is described as 0-order SI. In compliance with the symmetry law to achieve maximum symmetry, the OI on the entangled ring should have the same status as the 0 order SI, so it is considered that the OI ring also occupies the 0 order orbit as shown in Figure 9. When OI leaves the "furthest point" and orbits on the ring, it would leave a gradually expanding wavy line at outside. The trace left by OI of SU as it passing the "apogee" is named as the object orbit. Since OI is infinitesimal the object orbits it left should also be infinitely narrow, so it can be presented with even number. Meanwhile, the entanglement of SUs in the ring would form common SI outside the ring in compliance with Eq.(9). On one hand, the density of common SI decreases following the increasing of distance to the ring. On the other hand, the OI rotates around the ring would leave an expanding object orbit and it would depict inhomogeneous space orbits. For the SI density of the space orbit is uneven, so they can be presented with odd numbers as shown in Figure 9 and 10.

The stable structure of OI in the outer ring and SI in the inner ring is the basis of the odd SI and even OI outside the ring. The most basic matter unit with static mass can be named as 1 grade steady state object-space unit (1 grade steady unit for short). The photon unit without mass, in which OI and SI 
dynamically alternate in the outer ring, can be named as the 1 grade dynamic object-space unit (1 grade dynamic unit for short). The odd number SI outside the 2-D ring and the 0 order SI inside the ring of the 1 grade steady unit constitute the odd- 0 composite space orbit. As a whole, the gravitational field is assumed to be the sum of all the "outside odd-inside 0" asymmetric composite space orbits outside the OI ring.

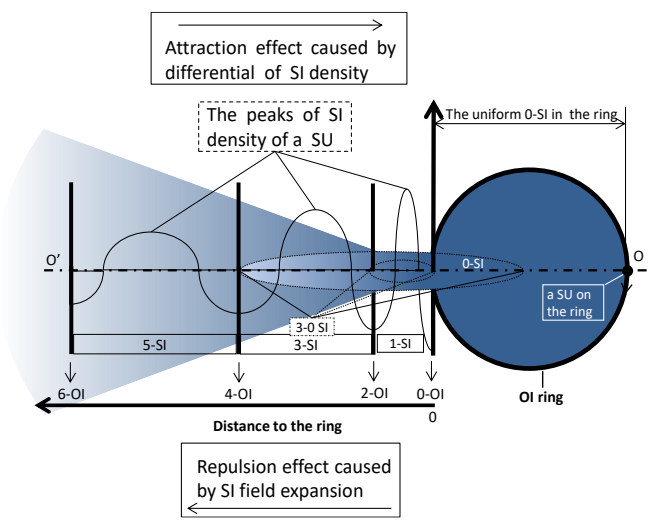

Figure 9: The asymmetric gravitational space field of 1 grade steady state unit[4]

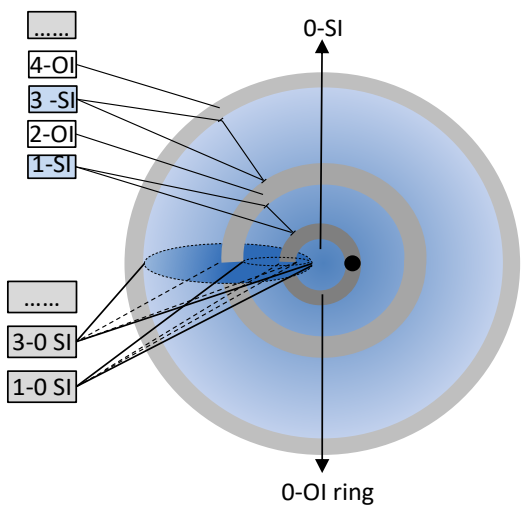

Figure 10: The structure of 1 grade steady state unit[4]

The process of gravitational action is generally as follows: Supposing that A and B both are steady state units, for the SI density outside the ring decreases following the farther distance to the ring, so the asymmetric of gravitation field is inversely proportional to the distance to the ring. In compliance with the symmetry law, the system tends to achieve maximum symmetry. When B is located in the odd space field of $\mathrm{A}$, the 0 -order $\mathrm{SI}$ of $\mathrm{B}$ inside its ring can compensate for the asymmetry of the complex (0-odd) SI orbit of A. Meanwhile, the 0 -order SI in the ring of A can also compensate for the SI asymmetry of B. Because the asymmetry of them both has the tendency of centripetal increasing, the closer the two matter rings are to each other before contact, the greater the compensatory effect of asymmetry is. Therefore, they would act as the universal law of gravity $\left(F=G M m / R^{2}\right)$.

Specifically, as the SI of a 2-D ring is distributed only on the 2-D plane outside the ring where it is located, when the density of the stationary 0 -order SI is $\rho_{0}$, then in compliance with the symmetry law, the sum of the SI density outside the ring is also $\rho_{0}$. Therefore, at the distance of $R$ from the ring center, its SI density $\rho$ should be $\rho_{0} /(2 \pi R)$. The reciprocity of gravitational action will make the strength of gravitational action inversely proportional to the square of the distance between the two parties, i.e. $F=\rho^{2}=\rho_{0}^{2} /\left(4 \pi^{2} R^{2}\right)$. It is consistent with the universal law of gravity $\left(F=G M m / R^{2}\right)$. Compared with the view on gravity of relativity, since the mass of the matter is equivalent to the 0 -order SI formed by the 2-D ring, and the asymmetry of the gravitational field is based on the 0 -order SI, so gravity cannot exist without mass. Accordingly, there is no flat time-space without mass as shown in Figure 1. On the other hand, the mass cannot exist independently from the asymmetric gravitational field, so the gravity and mass must depend on the finite large 2-D ring. Then the problem of gravitational field integral divergent can also be solved. What need to be emphasized is that the asymmetry of common SI is the basis of mass and gravity occurring. Or, there would be no mass, gravity field or any other energy fields exist. This is quite important for the unifying of gravity and electromagnetic forces.

Based on the above mechanism of gravitational action, it can be certain that, since accompanied with the constant mass of matter and the asymmetric gravitational space field formed by the entangled ring is infinite, then the mass-energy of the subject should not be lost to the gravitational field. In combination with the conservation principle of mass and energy, it can be known that the gravitational field should carry 0 mass or energy. Secondly, the ring supported by quantum entanglement is the most fundamental unit of mass and energy. The medium of electromagnetic force or the strong and weak forces are based on the collapse and exchange of the SU rings. As gravity action occurring there is no SU ring generation or collapse, so this can certify gravity is the only interaction between matters that does not have mass-energy medium exchange. This is the essential difference between gravity and the other three forces. Moreover, unlike the other three forces, which are based on conservation of energy and translational symmetry of space, there is a deeper conservation and symmetry behind the gravitational action, which is the conservation of symmetry and asymmetry. Specifically, gravitation only changes the distribution of symmetry and asymmetry between different areas of the universe.

In other words, there are not the so-called high energy gravitons that the quantization process of general relativity assumes to exist. Because the subject of gravitational field is a 2-D ring, when two black holes with astronomical multi-hierarchies ring suddenly merge to form a new black hole, their outer SI will inevitably undergo a ripple change. So this gravitational mechanism does not violate the fact that LIGO can detect gravitational waves when black holes collide. However, the existence of space ripples does not mean the existence of gravitational waves or gravitons with mass energy.

When taking for granted that gravity must also have intermediate as the other three forces, and tried to quantization analysis on the infinite gravitational field, the product of high-energy gravitons with infinite gravitational field certainly can't get converge integration results just as the other three fundamental forces. Therefore, it should be argued that the reason why general relativity fails to describe gravity in a perfect quantized way is that it does not properly understand the non-mediate form of gravity. Through deeper analysis, it can be concluded that the proposed mechanism of 
asymmetric gravitation under the framework of the symmetry law also lays a foundation for the unification of gravitation with electromagnetic force, and even all the four basic forces.

\section{Dark Matter and Dark Energy Mechanism}

The phenomenon of dark matter and dark energy are the two major problems associated with gravity in current physical theory field. Obviously, if these two problems can be reasonably explained under the framework of the proposed gravity scheme, it will undoubtedly be a strong proof of the correctness of the gravity scheme.

\subsection{Gravity Phenomena of Dark Matter}

An observation in 1970 by Vera Rubin on the stars rotation in the Andromeda Nebula found that the stars were moving much faster around the core than predicted $\left[{ }^{9]}\right.$. According to Newton's law of gravity, the gravitational centripetal force provided by the visible mass of the galactic nucleus decreases as the distance increases, and the corresponding velocity of stars in the galaxy's periphery decreases as well. However, it is observed that the velocity of the stars in the galaxy's periphery is constant over a large area, as shown in Figure 11. This means that there may be invisible dark matter in the galaxy's core that is far more massive than the total mass of the luminescent stars, providing extra mass than the visible matter. This is where the concept of dark matter comes from. So far, however, no evidence of dark matter has been found. In view of this, the author insists that dark matter maybe does not exist at all, and the phenomenon may simply be caused by the lack of proper mass and gravitational mechanism.

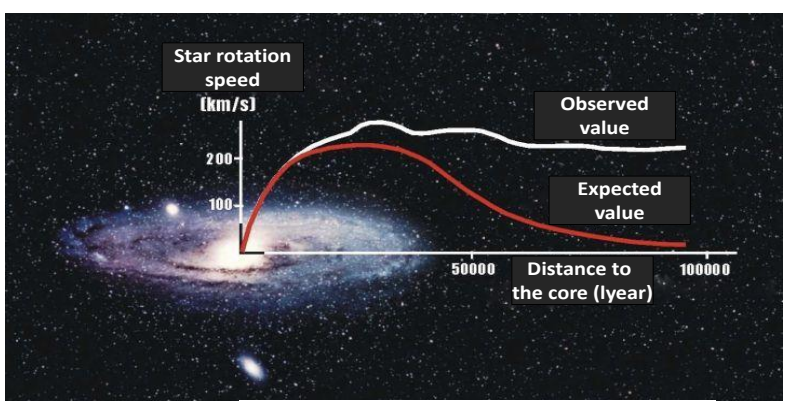

Figure 11: Gravitational effect of dark matter

According to Eq.(9) and symmetry law, the ring formed by SU will also be mapped and entangled with other SU when moving in space and form higher grades of ring. Combined with the analysis of the mechanism of 3-D twist ring of photon, the formation of higher grade of the 2-D ring is the basis of guaranteeing the stable 2-D ring existence. Theoretically, infinite grades of ring with mass and gravitational effect can be formed, but not be limited in the visible matters which actually is electromagnetic effect in nature. Different from the entangled rings formed by macroscopic moving matter, which are symmetrically distributed in different planes based on the symmetry law (this is the origin of constant velocity of photon), when the actual trajectory of matter is closed due to the gravitational action with the matter in the core, the equivalent mass and corresponding gravitational effect of all grades ring will play obvious mass and gravitational effect. This should be the origin of dark matter phenomenon found by Vera Rubin. This inference can be supported by phenomena such as mass-energy equivalence equation and electron wave-particle duality .etc.

This deduction enlarged the boundary of physical beings with gravitational field and mass effect. In other words, the mass and gravitation effect should not only be bound by the form of the visible matter. The visibility of matter is only a demonstration of having electromagnetic effect, but should not be the boundary of carrying mass and gravitation effect. To be certain, the visibility of matter is defined on the basis of its photonic properties capable of emitting or reflecting electromagnetic waves, while mass is defined on the basis of the gravitational and inertial effects of the entangled ring motion of matter. It's a question of two different kinds and grades of forces. That is to say, if SUs totally constitute a matter object of $n$ grades, the total mass effect of the $n$ grades of ring in huge enough observing scale is $\mathrm{A}$. In the $\mathrm{n}$ grades only $\mathrm{m}$ grades of ring have electromagnetic effect. Then, the mass of the visible substance B must be lower obviously than A. A may be several times of B if the observing scale is huge enough in the universe. For instance, according to measurements, the quarks that make up the proton make up only $7 \%$ of the total mass of the proton, and the gluons that interact with the quarks should have no mass as bosons, so the remaining $93 \%$ is the mass contained in the quark's higher-grade entangled ring in the scheme of this theory. This inference offers an available answer to the gravitational phenomenon of dark matter.

The following synthesis of the mass energy ring scheme and an important consequence of relativity is another strong support for the above explanation of the gravity of dark matter. It is well known that relativity dictates that no matter with mass, even the smallest, can travel at the velocity of photon (c). As they're accelerated to more approach to " $c$ ", they would absorb more energy. According to the mass-energy equivalence, the increase of energy actually is accompanied with the increase of relativistic mass. Fatherly, in order to more approach to $c$, more energy needs to be absorbed and until an infinite amount of energy is needed to bring it closer to the speed of photon. Finally, matter will have infinite mass but the velocity still can't be $c$. Relativity deduced from this that no matter with mass can reach the speed of photon at all (experimentally confirmed). However, relativity does not solve the problem of how energy behaves as mass. When the above process is combined with the understanding that both mass and energy are essentially entangled rings as this theory, it is easy to interpret it as following: taking the most basic mass unit, a steady-state unit of 1 grade for instance, in order to make its speed closer to c, it is necessary to continuously feed energy to it, which means the increasing on equivalent mass. Under the premise that there is only one core that is 1 grade of ring matter unit, combined with the nature of energy and mass both are entangled rings, it is obvious that the essence of mass energy increasing is the increasing on the grades of the ring. The quality provided by 1 grade of ring is only a small fraction of the total quality provided by all the nested grades of ring.

Returned to look at the unusually phenomenon of galaxies rotate caused by dark matter quality, due to the outer galaxies is rotating around the inner galaxy, along with a variety of rotation just as the moon around the earth, the earth moves 
around the sun, and the sun moves around the galactic core, at the same time the earth rotates to itself. According to the law of universal gravitation, the attraction of the moon to the sun is based only on the distance between them and the masses of them, while ignoring the gravitational and mass effects of each grade of SU ring based on all of the rotation. In this space and mass scale the mass maybe negligible. However, the total mass effect becomes completely non-negligible when we observing the distant galaxies. Like the invisible mass of proton and quarks, the mass and gravitational effect of these rotations may be several times greater than the mass and gravitational effect of visible matter. Therefore, according to this theory, the fundamental reason for the gravitational phenomenon of dark matter is the increasing on the grades of ring of the peripheral galaxies.

\subsection{Discussion on the Mechanism of Dark Energy Phenomenon}

Modern astronomy's observations of distant supernovae show that the universe is expanding, and the farther away the faster it expands[10,11]. According to Einstein's equations of gravity, the acceleration is believed to be caused by the existence of "dark energy" with negative pressure in the universe. However, up to now there is still no any evidence of dark energy has been found. In fact, it should be said that the way dark energy proposed is also seriously unreliable. According to the asymmetry gravitational mechanism, the author believes that the expansion of cosmic space is not caused by dark energy, but by the quantization with expansion feature of the gravitational field in the super-large cosmic scale.

According to the gravitation scheme shown in Figure 12, it can be inferred that since the outer SI is formed by the OI ring entanglement, the periodic and repeated rotation of single OI around the ring will make the gravitational field has the "three features" of quantization, spiralization and expansion as shown below:

(1) Quantization: the OI orbits left in the SI outside the ring by the OI rotating around the ring have the function of dividing the SI with quantized feature. Accordingly, the compound gravitational space field composed of odd SI and 0 order SI in the ring becomes a quantized field. Of course, the infinitely narrow of the OI orbit makes the quantized feature of the gravitational field very subtle. Furthermore, in the matter composed of a large number of steady-state units, the OI orbits are also covered by the SI of other units, which makes it difficult to show the quantized features of the gravitational field.

(2) Expansion: the repeated motion of the OI around the ring will constantly generate new object orbits and accordingly quantized space orbits. In addition, with the increasing of distance to the OI ring, the width of space orbit increases correspondingly. Therefore, the external SI field will expand outwards dynamically, and its expansion rate will accelerate with the increase of distance. This trend is same with the observed expanding trend of dark energy phenomenon.

(3) Spiralization: the directional rotation of SU on the ring also enables the external OI and SI the features of spiral due to the inevitable existence of the second grade of ring as shown in Figure 5-1. Combined with the analysis of the mechanism of photonic no static mass, the existence of a 2-grade of ring is the guarantee for the 1-grade closed ring in 2-D. On the other hand, the 2-grade ring would also rotate only if the energy is enough. Following the rotation of the 2-grade ring the spiral structure of the SI peak of the 1-grade ring would be formed. Based on the maximum compensation of asymmetry of SI, then stars would distribute in form of spiral structure as we've found in all galaxies.

According to above three features of gravitational field, obviously in a limit space scale, the distribution of less mass matter objects is much crowded than the matter objects with bigger mass. For instance, in the Milky Way, there are $2 \times 10^{11}$ stars totally, but only one main supermassive black hole (Sagittarius A at the galaxy's core). The spiral distribution of the Milky Way is mainly decided by it and a medium-sized black hole.

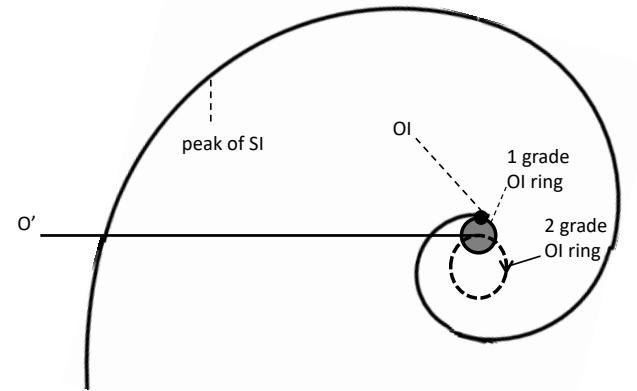

Figure 12: The expanse and spiral of space peak created by the 2 grades of OI ring

When the distribution density of matter objects is large, the three features of their gravitational fields will be covered by each other. Actually, since the volume of OI of SU is infinitesimally, the OI orbit of 1 -grade OI ring is correspondingly infinitely narrow in the outside. Therefore, even if the OI orbits of the 1-grade steady unit had the three features and they were not covered by others, they would also not have actual performance. But the most advanced matter objects, especially black holes at the cores of giant galaxies, the entangled rings of which have specific width, the three features of its gravitational field would show obvious and important influences inevitably then. In order to maintain the stable existence of the 1-grade ring, the black hole in the core of the galaxy also needs to make a further 2-grade ring movement, which will also make its outer SI form a spiral structure peak of asymmetry SI with outward accelerating expansion as shown in Figure 12. For the stars can make up for the asymmetry of SI to the greatest extent as they located at the peak, thus the external stars would distribute in form of spiral around the core.

When the distance to the core of the galaxy is far enough, with the weakening of the gravitational field of the low-mass star, only gravitational influence of the maximum object the black hole will be left. Then, the expansion and quantization features of the black hole gravity will be expressed. Since other galaxies would have the greatest effect of making up the asymmetry as they are located at the peak of SI, and then the attraction resulted from the SI density difference is negligible. Therefore, they would not be attracted closer to the galaxy now, but will be repelled by the accelerated expansion following distance increasing to the core of the SI 
peak. This is the origin of the so-called dark energy effect of the expansion of intergalactic space from the symmetry gravitation theory.

Moreover, according to the traditional concept of gravity, no matter how far away galaxies are, there is only gravity which attract them closer to each other. One day in the future, under this deducing direction, all galaxies in the infinite universe will gather into an infinite galaxy or even a black hole in the end, which is unreasonable obviously. Maybe someone would say then it's time for big bang happening of universe. But why we are so lucky to live at the time after big black hole and big bang? And where the energy comes from of the big bang? According to the gravity scheme in this paper, it is certain that there would be gravitational effect of matters at a limited space scale and there would be accelerated spatial repulsion effect at a sufficiently huge scale. In other words, galaxies mass would not increase unlimitedly and the universe would also not become more and more rare unlimitedly. The two opposite effect maintain the steady state of the universe. This laid the foundation for the existence of a steady-state universe with no beginning and no end.

\section{Analysis on Photon and the Unity of Locality and Nonlocality}

\subsection{The Source of Photon Non-static Mass Performance}

String theory uses finite large closed strings to solve the problem of integral divergence of gravitational field, but it does not attribute the formation of mass and gravity to the closed form of strings, which makes it impossible to explain the 0 static mass of photon. To this end, the theory was upgraded to superstrings theory in multidimensional space. This phase would explain the massless behavior of photon, but it would also lead to a series of super symmetric particles that should have been discovered under current technology, but up to now no any evidence have been found yet. This means that superstring theory is still failure and so it is reasonable to believe that the string theory is in the incorrect direction.

According to the analysis and reasoning of asymmetric gravity and mass generation under the framework of symmetry law, the existence of 2-D closed entangled ring is the key to the formation of static mass and gravitational field. As the unit with static mass, the closed ring on 2-D plane is formed under the supportion of the second grade or even more grade of ring, thus in the absence of second grade ring support, the photon can't form 2-D closed ring in any plane. As a twist closed ring, internal SI of photon is not completely orbited by the OI steadily, but following the constant macro motion of the photon, in compliance with the symmetry law the OI and SI dynamically orbit around each other. Accordingly, its SI around the ring can't form SI orbits with asymmetry just as the steady state unit with 2-D closed ring. So the photon does not have the performance of static mass and gravitational field is only resulted from its lack of 2-D closed ring.

What need to be highlighted is, just as the SI of relative static and motion SU having different expressions, the space and object structure of relative static and motion state photon are also different. Though actually the relative static photon is a twisted closed ring in 3-D, but as it moving forward its structure would be similar as the double helix structure of DNA. Figure 13 is the approximate relative moving structure of photon which is developed from the classical electromagnetic wave figure of photon. In the figure, its $\mathrm{OI}$ is presented as black circle and in order to show the symmetry feature its SI is also presented in white circle. The double helix alike structure is approximately discribed on two orthogonal planes and its not discribed as closed ring.Accurately the SI and OI of photon should not be in two standard orthogonal planes but a twisted closed ring in 3-D. In addition, it should be said that even in the photon the laws of electromagnetic induction is working also.

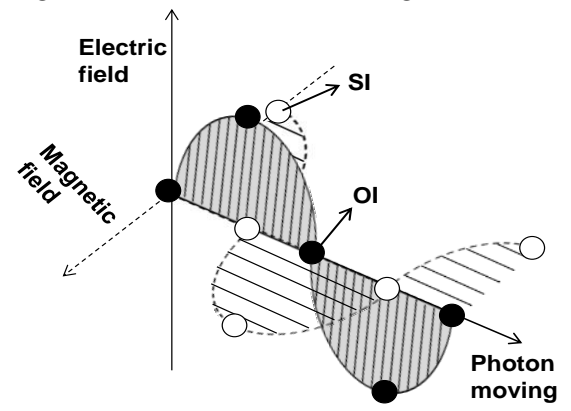

Figure 13: The dynamic symmetry photon[5,7]

\subsection{The Origin of Constant Velocity of Photon}

The constant velocity of photon is the basis of the theory of relativity, and it also has withstood a lot of practical examinations. Then what is the mechanism that causes this strange phenomenon which obviously violates the locality law? The author proposes that the answer to this question also lies behind the fact that the matter with massive mass would also create entanglement rings field as they moving. It means that, except microscopic particles such as photon and electron etc., the matter with massive mass, such as the illuminant, should also form entangled ring field as they moving. This ratiocination meets above interpretation of dark matter and the truth of equivalence of mass and energy. It would also be inferred then that the constant velocity should be a common phenomenon of all macroscopic moving matters.

According to the equivalent analysis on the special relativity by this theory, the event of the photon emitted from source to observer can be described by the product of the relative space distance $(S)$ and the relative SI density $(\rho)$ as $S \rho$. In other words, the photon from relative static and relative moving of illuminant to the same observer is equivalent, i.e., $S_{v} \rho_{v}=$ $S_{0} \rho_{0}=1 /(2 \pi)$. Based on this premise, then the increasing of the relative spatial distance $\left(S_{v}\right)$ can be proved is caused by the existence of the velocity $(v>0)$ as follows:

$$
\mathrm{V}>0 \stackrel{\rho_{v}=\rho_{0}\left(1-\frac{v^{2}}{c^{2}}\right)^{1 / 2}}{=} \rho_{v}<\rho_{0} \stackrel{s_{v} \rho_{v}=s_{0} \rho_{0}}{\Longrightarrow} S_{v}>S_{0}
$$

Above inference is on the basis of locality feature of illuminant motion. The reverse reasoning of the above process should also be reasonable. That is, if the illuminant motion also has the uncertainty and nonlocality nature just like electron or photon to the observer, then the relative distance of illuminant to observer would be equivalent to the distance of relative static of illuminant to observer $\left(S_{v}=S_{0}\right)$. Specifically, because it should be inferred that following with illuminant 
moving relatively to the observer, the entangled ring field covering observer would also be formed just as photon and electron motion, which supports the uncertainty of illuminant on the entangled ring. Alikely with uncertainty of photon and electron, the uncertainty of macro matter like illuminant would also have entangled mapping images everywhere on the rings. Though from Figure 4 it can be found the motion of illuminant would make the SI density at observer site decrease. However, the existence of illuminant mapping images on the ring, which have SI generation ability, would make up the decreasing of SI density. That's to say, the relative motion of the illuminant would not change its relative SI density to the observer. The space density of relative moving always equals to the relative static space density $\left(\rho_{v}=\rho_{0}\right)$. This is accordance with below reference. Combined with the relation of $S_{v} \rho_{v}=S_{0} \rho_{0}$ derived from the symmetry law, and the relation of $S_{v}=S_{0}$ derived from uncertainty feature, the relation of $\rho_{v}=\rho_{0}$ could also be gained. It means that, from the uncertainty nature of illuminant motion, three conclusions can be gained as below: the relative distance of photon passed with illuminant moving is equivalent to the distance of relative static state, the relative space density of motion state is equivalent to the static state resulted from the space density generation of entangled mapping images, and the velocity ( $v$ ) of the illuminant relative to the observer is equivalent to 0 . Therefore, the constant velocity of photon independent from motion velocity of any reference systems can be gained. It satisfies the following derivation process:

$$
S_{v}=S_{0} \stackrel{s_{v} \rho_{v}=s_{0} \rho_{0}}{\Longrightarrow} \rho_{v}=\rho_{0} \stackrel{\rho_{v}=\rho_{0}\left(1-\frac{v^{2}}{c^{2}}\right)^{1 / 2}}{=} \mathrm{V}=0
$$

From above analysis it also can be concluded that the constant velocity of photon is directly related to the unity of locality and nonlocality, but not a premise unnecessary to be explained as relativity assumed. And, the constant velocity is not the only feature of photon but of all moving matters which originally assumed to be locality.

\subsection{Unity of Locality and Nonlocality}

Through the successfully explanation on the dark matter and constant velocity of photon, it can be concluded that the assumption of macro matter motion will form entanglement ring field as micro particles is reasonable. At the same time, it also provides a solution to the dispute between locality and nonlocality. To be specific, locality is a false appearance based on the super mass of the moving subject, which conceals the existence of the entangled ring field and the nonlocality nature of them. The nonlocality based on the entangled ring is more essential, which is the universal existence of everything in the universe. These phenomenons of wave-particle duality, dark matter and constant velocity of photon all are resulted from this root cause.

What need to be analyzed more is about the relative space transformation process in relativity. It can be found that only if considering the existence of the constant velocity of photon and dealing with illuminant motion with locality precondition, the transformation of relative space, time and even the space density could be gained. Since it has been proved in practice that the velocity of photon is constant, and it cannot be reasonably explained in the framework of locality, then it is inevitable conclusion that the motion of macro matter having nonlocality nature. Once taking the nonlocality caused by entangled ring field of illuminant into consideration, the transformation of space, time and space density all would be unable. Therefore, it should be said that the sum of both taking locality representation of illuminant motion and nonlocality nature into consideration is the root to get special relativity results. However, the unclear on the mechanism of the constant velocity of photon and its nonlocality nature concealed the fact that special relativity actually had already accepted the existence of nonlocality in macro matter motion. From this point of view, the constant velocity of photon is also a powerful evidence for the inference of dark matter and wave-particle duality phenomenons.

\section{Summary}

Through the confirmation on the fundamental role of the symmetry law and the equivalent interpretation on the special relative theory without time dimension, this theory offered a self-consistent scheme in which the mechanism of gravity and mass, the phenomenon of dark matter and dark energy, the no static mass and constant velocity of photon, the unity of locality and nonlocality, are successfully incorporated. Furthermore, under this framework, it was found that the unified and consistent interpretation on the four fundamental forces of gravity, electromagnetic force, strong and weak force is also available. Therefore, in great extent the theory direction is correct.

\section{References}

[1] Jiao Gangzhen. Research on the existence form of gravitons and unified field theory based on the modification of relativistic space-time view[J]. Scientific Chinese, 2015, 14: 290-321.

[2] Jiao Gangzhen. Discussion on the nature of time based on relativity and the law of uncertainty[J]. Journal of Tianjin Polytechnic University, 2020, 36(1): 46-53.

[3] Natalie Wolchover. Why gravity is not like the other forces[E]. Quantum (Magazine), 20200615.

[4] Jiao Gangzhen. Discussion on mechanism of gravity on basis of Standard Ruler Nature of Time[J]. Journal of Tianjin Polytechnic University,2020. 36(3):53-58.

[5] Jiao Gangzhen. Interpretation of electromagnetic action by asymmetric mechanism[J]. Journal of Tianjin Polytechnic University, 2021, 36(1): 51-57.

[6] Pauli W (translated by Ling D H, Zhou W S). Theory of Relativity (in Chinese)[M]. Shanghai: Shanghai Science and Technology Press, 1979.

[7] L.I. Schiff. Quantum mechanics[M]. 3rd ed., New York and London: McGraw-Hill Book Company, 1968.

[8] Lee K C, Sprague M R, Sussman B J, et al.. Entangling macroscopic diamonds at room temperature $[\mathrm{J}]$. Science, 2011, 334(6060): 1253.

[9] Dewitt B S. General Relativity-An Einstein Centenary Survey[M]. Cambridge University Press, 1979.

[10] Wright, E.L.. What is the evidence for the Big Bang?. 2009, Frequently Asked Questions in Cosmology. UCLA.

[11] Penrose, R.. Difficulties with inflationary cosmology ${ }^{\mathrm{a}}[\mathrm{J}]$. Annals of the New York Academy of Sciences, 1989, 571(1): 249-264. 